'S Yurttutan, 'R Ozdemir, 'FE Canpolat, 'MY Oncel, ${ }^{2} \mathrm{HG}$ Unverdi, ${ }^{3} \mathrm{~B}$ Uysal, ${ }^{1} \mathrm{O}$ Erdeve, 'U Dilmen. 'Neonatal Intensive Care Unit, Zekai Tahir Burak Maternity Teaching Hospital; '2Department of Pathology, Ankara Etlik Ihtisas Training and Research Hospital; ${ }^{3}$ Department of Physiology, Gulhane Military Medical Academy, Ankara, Turkey

Introduction The pathophysiology of necrotizing enterocolitis (NEC) includes massive production of endogenous cytokines with exaggerated activation of inflammatory pathways. Colchicine has been used as an anti-inflammatory agent. The aim of this study was to investigate the possible beneficial effects of colchicine in a neonatal rat model of NEC.

Methods Rats were randomly divided into 3 groups: control group; saline-treated NECgroup; colchicine-treated NEC group. NEC was induced by hyperosmolar enteral formula feeding and exposure to hypoxia/reoxygenation after cold stress. Intestinal samples were harvested for biochemical and histopathological analysis. Results The grade of intestinal injury of pups in the saline-treated NEC group was found to be significantly higher than in the control or the colchicine-treated groups $(p<0.001, p=0.003$; respectively). Median level of intestinal malondialdehyde was significantly higher in the saline-treated NEC group compared to the control group $(p=0.006)$ and the colchicine-treated group $(p=0.015)$. Significantly higher activities of intestinal superoxide dismutase and glutathione peroxidase activities were observed in the colchicine-treated NEC group compared to the saline-treated group $(p=0.033$ and $p=0.030$; respectively). Tissue levels of tumor necrosis factor- $\alpha$ and interleukin $1 \beta$ were significantly higher in the saline-treated NEC group compared to rats in the colchicine-treated group $(p<0.001, p=0.003$; respectively). A comparison of saline-treated and colchicine-treated NEC pups revealed that treatment with colchicine was associated with significantly lower tissue levels of TNF- $\alpha$ and IL-1 $\beta$ ( $p<0.01$, both).

Conclusion We observed that; in this model of NEC, colchicine has favorable effects onintestinal histological and biochemical changes.

\section{SYSTEMATIC REVIEW OF RANDOMIZED CONTROL TRIALS TO REVIEW THE ROLE OF PREBIOTICS IN PREVENTION OF NECROTIZING ENTEROCOLOITIS IN PRETERM NEWBORNS}

doi:10.1136/archdischild-2012-302724.0187

'S Ali, ${ }^{2} \mathrm{~L}$ Faruque, ${ }^{3 \mathrm{M}}$ Kumar. ${ }^{1}$ Neonatal Perinatal Medicine, Department of Pediatrics, University of Alberta; ${ }^{2}$ Epidemiology; School of Public Health; ${ }^{3}$ Department of Pediatrics, University of Alberta, Edmonton, $A B$, Canada

Background and aims Necrotizing enterocolitis (NEC) is an important cause of mortality and serious morbidity in preterm infants. Prebiotics are specific oligosaccharides which have been shown to promote proliferation of beneficial bacteria in gut. This systematic review aims to review the literature to investigate the role of prebiotics in the prevention of NEC in preterm infants.

Methods Electronic databases CSDR-DARE, MEDLINE, CINAHL, EMBASE, Scopus, Web of science were serached from the date of inception to March 27, 2012. Additional citations were retrieved from the bibliography of the selected articles, Google scholarand abstracts of conference proceedings. The eligible studies were RCTs or quasi-RCTs enrolling inpatient preterm infants that compared use of Prebiotics (any dose and duration) with control (placebo/no treatment) for the outcomes of NEC (stage $>=2$ Bell's classification, perforation and any stage), growth and any other potentially beneficial effect or serious side-effects. Two independent reviewers extracted the data and assessed the risk of bias in included studies. Descripencies were resolved with consensus.

Results 14 studies fulfilled the inclusion criteria. None reported on the primary outcome of stage $>=2$ NEC. Two RCT reported on NEC (any stage) and showed no significant difference between the groups. There was no difference noted in the growth parameters [(weight \& length (3 studies); head growth (2 studies)]. There was a trend towards higher stool frequency (one study) and higher Bifidobacterium count in stool (2 studies) in the Prebiotic group.

Conclusion Current data is insufficient to recommend the use of Prebiotics in preterm infants for prevention of NEC.

\section{ANALGESIA AND SEDATION IN CRITICALLY ILL CHILDREN; LOCAL RELIGION OR EVIDENCE BASED?}

doi:10.1136/archdischild-2012-302724.0188

D Tibboel. Pain Research Team, Erasmus MC - Sophia Childrens Hospital, Rotterdam, The Netherlands

Over the last decade increasingly RCT's have been published about optimal dosing of opioids and benzodiazepines in critically ill children of different age groups.

In this way progress is made about optimal dosing as well as combination of therapies against the background of the use of novel ways of trial design.

To this effect the application of population pharmacokineticspharmacodynamics (NON-MEM) using spare data have guided the design of trials preceded by in vitro simulation and prediction of dose effect responses.

Both in the premature infant as well as in the so-called surgical newborn, dosages have been adjusted based on solid observational and experimental data sets for which the results should be evaluated. Apart from short term pharmacodynamic parameters such as validated pain scores, and eventually pharmacokinetic data analysis potentially equally important is the evaluation of long term consequences both of neonatal pain and the use of opioids. Experimental data have revealed increased neuro apoptosis in the developing brain. The data of a number of RCT's conducted by our group will be combined with prospective longitudinal data recently acquired combining quantitative sensory testing (OST) under conditions of fMRI.

In this way the question whether neonatal pain and/or opioid use results in altered pain response and long term negative sequelae can be answered.

\section{STILL HURTING NEWBORN BABIES EIGHT YEARS AFTER WE FOUND OUT!}

doi:10.1136/archdischild-2012-302724.0189

${ }^{1} \mathrm{D}$ Roofthooft, 'S Simons, ${ }^{2} \mathrm{~K}$ Anand, ${ }^{3} \mathrm{D}$ Tibboel, ${ }^{3} \mathrm{M}$ van Dijk. ${ }^{1}$ Neonatology, Erasmus MC - Sophia Children's Hospital, Rotterdam, The Netherlands; 2 Pediatrics, University of Tennessee Health Science Center ${ }^{*}$ Le Bonheur, Memphis, TN, USA; Intensive Care, Erasmus MC - Sophia Children's Hospital, Rotterdam, The Netherlands

Objective To study whether new pharmacological and non-pharmacological guidelines lowered numbers of painful procedures in neonates and changed the amount and frequency of analgesic therapy as compared to the results of our previous study in 2001.

Design A prospective observational study.Setting: Level III NICU of the Erasmus MC-Sophia Children's Hospital, Rotterdam.

Participants Neonates admitted at postnatal ages less than 3 days with length of stay at least 72 hours

Main outcome measures Number of all potentially painful procedures and analgesic therapy recorded at the bedside during the first 14 days of NICU stay.

Results A total number of 21076 procedures were performed in the 175 neonates studied during 1730 patient-days (mean 12.2). The mean number of painful procedures per neonate per day was 11.4 (SD 5.7), significantly lower than the number of 14.3 (SD 4.0) in 2001 ( $p<0.001$ ). The use of analgesics was $36.6 \%$ compared to $60.3 \%$ in 2001. Failed procedures encompassed sixty-three percent of all peripheral arterial line insertions vs. $37.5 \%$ in 2001 and $9.1 \%$ venipunctures vs. $21 \%$ in 2001 . 